International Journal of Pure and Applied Mathematics

Volume 92 No. 5 2014, 619-644

ISSN: 1311-8080 (printed version); ISSN: 1314-3395 (on-line version)

url: http://www.ijpam.eu

doi: http://dx.doi.org/10.12732/ijpam.v92i5.1

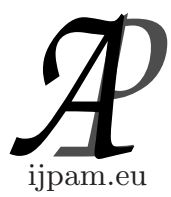

\title{
EXPONENTIAL SYNCHRONIZATION FOR HYBRID COUPLED NEURAL NETWORKS WITH TIME DELAYS VIA INTERMITTENT FEEDBACK CONTROLS
}

\author{
N. Yotha ${ }^{1}$, T. Mouktonglang ${ }^{2}$, T. Botmart ${ }^{3} \S$ \\ ${ }^{1,2}$ Department of Mathematics \\ Chiangmai University \\ Chiangmai, 50200, THAILAND \\ ${ }^{3}$ Department of Mathematics \\ Srinakharinwirot University \\ Bangkok, 10110, THAILAND \\ ${ }^{3}$ Centre of Excellence in Mathematics CHE \\ Si Ayutthaya Rd., Bangkok 10400, THAILAND
}

\begin{abstract}
This paper, investigates the exponential synchronization of neural networks with time delays and hybrid coupling composed of constant coupling, time-varying delay coupling and distributed delay coupling. The designed controller ensures the exponential synchronization of hybrid couple delayed neural networks are proposed via intermittence feedback control. Based on the design of the outer-coupling matrices, the inner-coupling matrix matrices, and some free matrices representing the relationships between the system matrices. Some sufficient conditions are established to achieve exponential synchronization by constructing of improved Lyapunov-Krasovskii functionals and Kronecker product properties. The criteria are derived in terms of LMIs which can be solved efficiently by standard convex optimization algorithms. A numerical example
\end{abstract}

Received: October 12, 2013

(c) 2014 Academic Publications, Ltd. url: www.acadpubl.eu

${ }^{\S}$ Correspondence author 
and its simulation are given to show the effectiveness of the obtained results.

AMS Subject Classification: 47H09, 47H10

Key Words: neural networks, exponential synchronization, time-varying delays, leakage delay, hybrid coupling

\section{Introduction}

During the last few years, the network synchronization has been widely investigated by reason its high potential application in various fields. The system is one of hot research fields which has been a challenging issue because it presents in many applications such as signal processing, associative memory, some engineering applications and so on $[1,2,3]$. Synchronization is two or more networks which are either networks or periodic share a common dynamical behavior and it has been appeared that this common behavior can be induced by coupling or by outer force $[1,4]$. Though the authors have made some initial efforts on constant coupling for such problem, the derived results [5] cannot tackle the variable delay, which means that their methods need great improvements. So, there exist coupling terms between the state variables and the delayed ones, in which the delay ones can explain the decentralized nature of real-word coupled networks. Therefore during considering the synchronization of networks, the constant coupling, delay coupling, and distributed delay coupling should be taken into discussion altogether, which led to the introduction of hybrid coupling $[6,7,8,9]$. In [9], the cluster synchronization in an array of hybrid coupled neural networks with delay has been studied which is shown that this common behavior can be induced by coupling in neural networks.

Neural networks can show some complicated dynamics and even chaotic behavior, the synchronization of chaotic neural networks has also become an significant area of study. Newly, the synchronization problems for neural networks also have been presented and there have been many investigations. In [10], the researcher has been shown for the synchronization in an array of linearly coupled networks with time-varying delay. It is well recognized that timevarying delay is frequently encountered in electronic implementations of neural networks due to the finite switching speed of the amplifiers and communication time. Therefore, it is necessary to consider the variable time-varying delay effect when investigating the synchronization problem of neural networks. Delayed neural networks were thus proposed and have got a great deal of attention $[9,10,11,12,13]$. On the other hand, a leakage delay, which is the time delay in leakage term of the networks and a considerable factor affecting dynamics 
for the worse in the networks $[13,14,15]$. However, NNs may not be synchronized when a controller is not added into the infrastructure of individual nodes. Hence the controlled synchronization of NNs is believed to be a rather important topic in both theoretical research and practical applications, such as sample-data control [16], adaptive control [17], feedback control [18], intermittent control [19], etc.

Intermittent control is to control systems via discontinuous control inputs at a control period $[19,20,21,22,23]$. In some cases, it can be impossible to use only synchronization of NNs at all times. In fact, to use synchronization of NNs in some intervals only may prove to be more cost effective than using synchronization of NNs at all times. Another reason could be that the intermittent control scheme might be useful in communications, where synchronization of NNs between transmitter and receiver can be used as means of transmitting information [20]. Hence, it is challenging to create some less conservative and easy-to-check criteria guarantee the exponential synchronization for hybrid coupled NNs with time delays delay via intermittent feedback controls, which constitutes the main focus of this propose work.

In this paper, we make the great efforts to investigate the exponential synchronization of neural networks with interval time-varying delays, leakage delay and hybrid coupling. The designed controller ensures the exponential synchronization via feedback control and intermittent feedback control. By constructing an augmented Lyapunov-Krasovskii functional and using appropriate integral inequality, two novel sufficient conditions are obtained in terms of LMIs. The feasibility of the conditions can be easily checked by resorting to Matlab LMI Toolbox. The new synchronization conditions are much less conservative and more general than some existing results $[9,13,14]$. Finally, the effectiveness of the proposed criteria can be demonstrated by utilizing three numerical examples.

The rest of this paper is organized as follows. In Section 2 we give notations, mathematical model of neural networks with time delays, assumption, definition and lemmas for using in the proof of the main results. The exponential synchronization problems are studied and some sufficient conditions are developed in Section 3. Numerical examples illustrated the obtained results are given in Section 4. The paper ends with conclusions in Section 5 and cited reference.

Notation $R^{n}$ is the $n$-dimensional Euclidean space; $R^{m \times n}$ denotes the set of $m \times n$ real matrices; $I_{n}$ represents the $n$-dimensional identity matrix; $\lambda(A)$ denotes the set of all eigenvalues of $A ; \lambda_{\max }(A)=\max \{\operatorname{Re} \lambda ; \lambda \in \lambda(A)\}$; $C\left([0, t], R^{n}\right)$ denotes the set of all $R^{n}$-valued continuous functions on $[0, t]$; 
$L_{2}\left([0, t], R^{m}\right)$ denotes the set of all the $R^{m}$-valued square integrable functions on $[0, t]$; The notation $X \geq 0$ (respectively, $X>0$ ) means that $X$ is positive semidefinite (respectively, positive definite); The notation $A \otimes B$ stands for the Kronecker product of matrices $A$ and $B ; \operatorname{diag}(\cdots)$ denotes a b lock diagonal matrix; $\left[\begin{array}{cc}X & Y \\ * & Z\end{array}\right]$ stands for $\left[\begin{array}{cc}X & Y \\ Y^{T} & Z\end{array}\right]$. Matrix dimensions, if not explicitly stated, are assumed to be compatible for algebraic operations.

\section{Preliminaries}

Consider the coupled dynamical system consisting of $N$ linearly and diffusively coupled recurrent neural networks with time delays can be described by:

$$
\begin{aligned}
\dot{y}_{i}(t)= & -C y_{i}(t-\sigma)+A f\left(y_{i}(t)\right)+B g\left(y_{i}(t-\tau(t))\right) \\
& +D \int_{t-k(t)}^{t} h\left(y_{i}(s)\right) d s+E U_{i}(t)+\sum_{j=1}^{N} g_{i j}^{1} \Gamma_{1} y_{j}(t) \\
& +\sum_{j=1}^{N} g_{i j}^{2} \Gamma_{2} y_{j}(t-\tau(t))+\sum_{j=1}^{N} g_{i j}^{3} \Gamma_{3} \int_{t-k(t)}^{t} h\left(y_{j}(s)\right) d s, \\
y_{i}(t)= & \phi_{i}(t), t \in\left[\tau_{\max }, 0\right], \tau_{\max }=\max \left\{\tau_{2}, k\right\}, i=1,2, \ldots, N,
\end{aligned}
$$

where $y_{i}(t)=\left(y_{i 1}(t), y_{i 2}(t), \ldots, y_{i n}(t)\right)^{T} \in R^{n}$ is the state vector of the $i-t h$ network at time $t . C=\operatorname{diag}\left(c_{1}, c_{2}, \ldots, c_{n}\right)>0$ denotes the rate with which the cell $i$ resets its potential to the resting state when being isolated from other cells and inputs, $A, B, D$ and $E$ represent the connection weight matrix, $G^{q}=g_{i j}^{q} \in R^{n \times n},(q=1,2,3)$ represent the outer-coupling matrix of the network, $\Gamma_{1}, \Gamma_{2}, \Gamma_{3} \in R^{n \times n}$ represent a constant inner-coupling matrix, $f\left(y_{i}(t)\right)=$ $\left(f_{1}\left(y_{i 1}(t)\right), \ldots, f_{n}\left(y_{i n}(t)\right)\right)^{T}, g\left(y_{i}(t-\tau(t))\right)=\left(g_{1}\left(y_{i 1}(t-\tau(t))\right), \ldots, g_{n}\left(y_{i n}(t-\right.\right.$ $\tau(t))))^{T}, h\left(y_{i}(s)\right)=\left(h_{1}\left(y_{i 1}(s)\right), \ldots, h_{n}\left(y_{i n}(s)\right)\right)^{T}$ represent the activation function vectors and $U_{i}(t) \in R^{n}$ represent the control input vector.

The interval time-varying delays, time delays and leakage delay are $\tau(t)$, $k(t)$ and $\sigma$, respectively, satisfy the following conditions

$$
\begin{array}{ll}
0 \leq \tau_{1} \leq \tau(t) \leq \tau_{2}, & \dot{\tau}(t) \leq \mu_{1}, \quad \eta=\tau_{2}-\tau_{1}, \\
0<k(t) \leq k, & \dot{k}(t) \leq \mu_{2}, \quad 0<\sigma .
\end{array}
$$

We denote the synchronization error by $x_{i}(t)=y_{i}(t)-\widehat{y}(t)$ where $\widehat{y}(t) \in R^{n}$ is the state trajectory of the unforced isolate node. Then, the error dynamics of 
network (1) can be obtained as follows:

$$
\begin{aligned}
\dot{x}_{i}(t)= & -C x_{i}(t-\sigma)+A f\left(x_{i}(t)\right)+B g\left(x_{i}(t-\tau(t))\right) \\
& +D \int_{t-k(t)}^{t} h\left(x_{i}(s)\right) d s+E U_{i}(t)+\sum_{j=1}^{N} g_{i j}^{1} \Gamma_{1} x_{j}(t) \\
& +\sum_{j=1}^{N} g_{i j}^{2} \Gamma_{2} x_{j}(t-\tau(t))+\sum_{j=1}^{N} g_{i j}^{3} \Gamma_{3} \int_{t-k(t)}^{t} h\left(x_{j}(s)\right) d s, \\
x_{i}(t)= & \phi_{i}(t), t \in\left[\tau_{\max }, 0\right], \tau_{\max }=\max \left\{\tau_{2}, k\right\}, i=1,2, \ldots, N,
\end{aligned}
$$

In this paper, we design the feedback controller $U_{i}(t), i=1, \ldots, N$ satisfy either $\left(\mathcal{U}_{1}\right)$ or $\left(\mathcal{U}_{1}\right)$ :

$$
\begin{aligned}
& \left(\mathcal{U}_{1}\right): U_{i}(t)=K x_{i}(t) \quad t \geq 0, \\
& \left(\mathcal{U}_{2}\right): U_{i}(t)=\left\{\begin{array}{ll}
K x_{i}(t), & n \omega \leq t \leq n \omega+\delta, \\
0, & n \omega+\delta<t \leq(n+1) \omega,
\end{array},\right.
\end{aligned}
$$

where $K$ is a constant control gain, $\omega>0$ is the control period and $\delta>0$ is called the control width (control duration) and $n$ is a non-negative integer.

With the Kronecker product (2), we can reformulate as follows:

$$
\begin{aligned}
\dot{x}(t)= & -\left(I_{N} \otimes C\right) x(t-\sigma)+\left(I_{N} \otimes A\right) f(x(t)) \\
& +\left(I_{N} \otimes B\right) g(x(t-\tau(t)))+\left(I_{N} \otimes D\right) \int_{t-k(t)}^{t} h(x(s)) d s \\
& +\left(I_{N} \otimes E\right) U(t)\left(G^{1} \otimes \Gamma_{1}\right) x(t)+\left(G^{2} \otimes \Gamma_{2}\right) x(t-\tau(t)) \\
& +\left(G^{3} \otimes \Gamma_{3}\right) \int_{t-k(t)}^{t} h(x(s)) d s,
\end{aligned}
$$

where $x^{T}(t)=\left[x_{1}^{T}(t), \ldots, x_{N}^{T}(t)\right], f^{T}(x(t))=\left[f^{T}\left(x_{1}(t)\right), \ldots, f^{T}\left(x_{N}(t)\right)\right]$, $g^{T}(x(t-\tau(t)))=\left[g^{T}\left(x_{1}(t-\tau(t))\right), \ldots, g^{T}\left(x_{N}(t-\tau(t))\right)\right]$, $h^{T}(x(s))=\left[h^{T}\left(x_{1}(s)\right), \ldots, h^{T}\left(x_{N}(s)\right)\right], U^{T}(t)=\left[U_{1}^{T}(t), \ldots, U_{N}^{T}(t)\right]^{T}$ and its identical Kronecker product from are

$$
\begin{aligned}
& \left(\mathcal{U}_{1}\right): \quad U(t)=\left(I_{N} \otimes K\right) x(t) \quad t \geq 0, \\
& \left(\mathcal{U}_{2}\right): \quad U(t)= \begin{cases}\left(I_{N} \otimes K\right) x(t), & n \omega \leq t \leq n \omega+\delta, \\
0, & n \omega+\delta<t \leq(n+1) \omega,\end{cases}
\end{aligned}
$$

Throughout this paper, we make the following assumptions: 
Assumption 1. The outer-coupling configuration matrices of the complex networks satisfy: $\quad g_{i j}^{q}=g_{j i}^{q} \geq 0, \quad g_{i i}^{q}=-\sum_{j=1, j \neq i}^{N} g_{i j}^{q}$. where $i, j=1,2, \ldots, N, \quad i \neq j$ and $q=1,2,3$.

Assumption 2. For any constants $\rho_{r}^{-}, \rho_{r}^{+}, \varrho_{r}^{-}, \varrho_{r}^{+}, v_{r}^{-}, v_{r}^{+}$, the function satisfy:

$$
\begin{aligned}
& \rho_{r}^{-} \leq \frac{f_{r}\left(s_{1}\right)-f_{r}\left(s_{2}\right)}{s_{1}-s_{2}} \leq \rho_{r}^{+}, \\
& \varrho_{r}^{-} \leq \frac{g_{r}\left(s_{1}\right)-g_{r}\left(s_{2}\right)}{s_{1}-s_{2}} \leq \varrho_{r}^{+}, \\
& v_{r}^{-} \leq \frac{h_{r}\left(s_{1}\right)-h_{r}\left(s_{2}\right)}{s_{1}-s_{2}} \leq v_{r}^{+},
\end{aligned}
$$

we denote

$$
\begin{aligned}
& F_{1}=\operatorname{diag}\left(\rho_{1}^{+} \rho_{1}^{-}, \cdots, \rho_{n}^{+} \rho_{n}^{-}\right), F_{2}=\operatorname{diag}\left(\frac{\rho_{1}^{+}+\rho_{1}^{-}}{2}, \cdots, \frac{\rho_{n}^{+}+\rho_{n}^{-}}{2}\right), \\
& J_{1}=\operatorname{diag}\left(\varrho_{1}^{+} \varrho_{1}^{-}, \cdots, \varrho_{n}^{+} \varrho_{n}^{-}\right), J_{2}=\operatorname{diag}\left(\frac{\varrho_{1}^{+}+\varrho_{1}^{-}}{2}, \cdots, \frac{\varrho_{n}^{+}+\varrho_{n}^{-}}{2}\right), \\
& L_{1}=\operatorname{diag}\left(v_{1}^{+} v_{1}^{-}, \cdots, v_{n}^{+} v_{n}^{-}\right), L_{2}=\operatorname{diag}\left(\frac{v_{1}^{+}+v_{1}^{-}}{2}, \cdots, \frac{v_{n}^{+}+v_{n}^{-}}{2}\right) .
\end{aligned}
$$

We introduce the following technical well-known Lemma, which will be used in the proof of our results.

Definition 1. Given $\alpha>0$. System (5) is said to be exponentially synchronized if there exist a positive number $M>0$ such that every solution $x(t, \phi)$ satisfies the following condition:

$$
\|x(t, \phi)\| \leq M e^{-\alpha t}\|\phi\|, \quad \forall t \geq 0 .
$$

Lemma 1. Let $\otimes$ denote the notation of Kronecker product. Then, the following relationships hold:

$$
\begin{aligned}
& \text { (1) }(\alpha A) \otimes B=A \otimes(\alpha B), \\
& \text { (2) }(A+B) \otimes C=A \otimes C+B \otimes C, \\
& \text { (3) }(A \otimes B)(C \otimes D)=(A C) \otimes(B D) .
\end{aligned}
$$

Lemma 2. [7] Let $e=(1,1, \ldots, 1)^{T}, E_{N}=e e^{T}$, and $U=N I_{N}-E_{N}, P \in$ $R^{n \times n}, x=\left(x_{1}^{T}, \ldots, x_{N}^{T}\right)^{T}$, and $y=\left(y_{1}^{T}, \ldots, y_{N}^{T}\right)^{T}$ with $x_{k}, y_{k} \in R^{n},(k=$ 
$1,2, \ldots, N)$, then

$$
x^{T}(U \otimes P) y=\sum_{1 \leq i<j \leq N}^{N}\left(x_{i}-x_{j}\right)^{T} P\left(y_{i}-y_{j}\right) .
$$

Lemma 3. [11] For any symmetric positive definite matrix $M>0$, scalar $\gamma>0$ and vector function $x:[0, \gamma] \rightarrow R^{n}$ such that the integrations concerned are well defined, the following inequality holds:

$$
\left(\int_{0}^{\gamma} x(s) d s\right)^{T} M\left(\int_{0}^{\gamma} x(s) d s\right) \leq \gamma\left(\int_{0}^{\gamma} x^{T}(s) M x(s) d s\right) .
$$

Lemma 4. (Cauchy inequality) For any symmetric positive definite matrix $N \in M^{n \times n}$ and $x, y \in R^{n}$ we have

$$
\pm 2 x^{T} y \leq x^{T} N x+y^{T} N^{-1} y .
$$

Lemma 5. [11] (Schur complement). Given constant symmetric matrices $X, Y, Z$ with appropriate dimensions satisfying $X=X^{T}, Y=Y^{T}>0$. Then $X+Z^{T} Y^{-1} Z<0$ if and only if

$$
\left(\begin{array}{cc}
X & Z^{T} \\
Z & -Y
\end{array}\right)<0 \quad \text { or } \quad\left(\begin{array}{cc}
-Y & Z \\
Z^{T} & X
\end{array}\right)<0 .
$$

\section{Main Results}

First, we present a delay-dependent stabilizability analysis conditions for the given nonlinear time-varying delay systems (5), and derive easy-to-verify sufficient conditions to guarantee the coupled system to be exponentially synchronized. Let us set

$$
\begin{aligned}
\rho_{r}= & \max \left\{\left|\rho_{r}^{-}\right|,\left|\rho_{r}^{+}\right|\right\}, \varrho_{r}=\max \left\{\left|\varrho_{r}^{-}\right|,\left|\varrho_{r}^{+}\right|\right\}, v_{r}=\max \left\{\left|v_{r}^{-}\right|,\left|v_{r}^{+}\right|\right\}, \\
\lambda_{1}= & \lambda_{\min }(P), \\
\lambda_{2}= & \lambda_{\max }(P)+\sigma \lambda_{\max }\left(Q_{1}\right)+\tau_{1} \lambda_{\max }\left(Q_{2}\right)+\tau_{2} \lambda_{\max }\left(Q_{3}\right) \\
& +\tau_{2} \lambda_{\max }\left(Q_{4}\right)+\varrho_{r}^{2} \tau_{2} \lambda_{\max }\left(Q_{5}\right)+\tau_{1}^{2} \lambda_{\max }\left(R_{1}\right)+\tau_{2}^{2} \lambda_{\max }\left(R_{2}\right) \\
& +\eta^{2} \lambda_{\max }\left(R_{3}\right)+\sigma^{2} \lambda_{\max }\left(S_{1}\right)+k^{2} \lambda_{\max }\left(S_{2}\right)+v_{r}^{2} k^{2} \lambda_{\max }\left(S_{3}\right) .
\end{aligned}
$$


Theorem 2. Given $\alpha>0$ and suppose that assumption 1-2 hold, the system (5) is exponentially synchronized under the controller (3) if there exist symmetric positive definite matrices, $P, Q_{k}, R_{l}, S_{l}, Y(k=1,2, \ldots, 5, l=1,2,3)$ and positive diagonal matrix $\Omega, \Theta, \Delta$ with appropriate dimensions, such that the following linear matrix inequalities (LMIs) hold for all $1=i<j=N$ :

$$
\begin{aligned}
& \Sigma_{1 i j}=\mathcal{M}_{i j}-\left[\begin{array}{llllllllllll}
0 & 0 & 0 & 0 & 0 & 0 & 0 & I & 0 & -I & 0 & 0
\end{array}\right]^{T} e^{-2 \alpha \tau_{2}} \\
& \times R_{3}\left[\begin{array}{llllllllllll}
0 & 0 & 0 & 0 & 0 & 0 & 0 & I & 0 & -I & 0 & 0
\end{array}\right]<0, \\
& \Sigma_{2 i j}=\mathcal{M}_{i j}-\left[\begin{array}{llllllllllll}
0 & 0 & 0 & 0 & 0 & 0 & 0 & -I & I & 0 & 0 & 0
\end{array}\right]^{T} e^{-2 \alpha \tau_{2}} \\
& \times \quad R_{3}\left[\begin{array}{llllllllllll}
0 & 0 & 0 & 0 & 0 & 0 & 0 & -I & I & 0 & 0 & 0
\end{array}\right]<0, \\
& \Sigma_{3}=\left[\begin{array}{ccc}
-0.1 e^{-2 \alpha \tau_{1}} R_{1}-0.1 e^{-2 \alpha \tau_{2}} R_{2} & 2 Y^{T} & P E \\
* & -2 P & 0 \\
* & * & -P
\end{array}\right]<0, \\
& \Sigma_{4}=\left[\begin{array}{cc}
-T & T E \\
* & -P
\end{array}\right]<0,
\end{aligned}
$$

where

$$
\begin{aligned}
& \mathcal{M}_{i j}=\left[\begin{array}{ccccc}
M_{1,1} & M_{1,2} & M_{1,3} & \cdots & M_{1,12} \\
* & M_{2,2} & M_{2,3} & \cdots & M_{2,12} \\
* & * & M_{3,3} & \cdots & M_{3,12} \\
\vdots & \vdots & \vdots & \ddots & \vdots \\
* & * & * & \cdots & M_{12,12}
\end{array}\right] \\
& M_{1,1}=-N g_{i j}^{1} P \Gamma_{1}-N g_{i j}^{1} \Gamma_{1}^{T} P+\sum_{r=1}^{4} Q_{r}-0.9 e^{-2 \alpha \tau_{1}} R_{1} \\
& -0.9 e^{-2 \alpha \tau_{2}} R_{2}-e^{-2 \alpha \sigma} S_{1}+k S_{2}-\Omega F_{1}-\Theta J_{1}-\Delta L_{1}, \\
& M_{1,2}=-e^{-2 \alpha \sigma} S_{1}+C^{T} P, \quad M_{1,3}=-N g_{i j}^{1} T \Gamma_{1}, \\
& M_{1,4}=B^{T} P, \quad M_{1,5}=\Omega F_{2}, \\
& M_{1,6}=\Theta J_{2}+A^{T} P, \quad M_{1,7}=\Delta L_{2}, \\
& M_{1,8}=-N g_{i j}^{2} \Gamma_{2}^{T} P, \quad M_{1,9}=e^{-2 \alpha \tau_{1}} R_{1}, \\
& M_{1,10}=e^{-2 \alpha \tau_{2}} R_{2}, \quad M_{1,12}=-N g_{i j}^{3} \Gamma_{3}^{T} P+D^{T} P \text {, } \\
& M_{2,2}=-e^{-2 \alpha \sigma}\left(Q_{1}+S_{1}\right), \quad M_{2,3}=-T C, \\
& M_{3,3}=\tau_{1}^{2} R_{1}+\tau_{2}^{2} R_{2}+\eta^{2} R_{3}+\sigma^{2} S_{1}-T, \quad M_{3,4}=B^{T} T \\
& M_{3,6}=A^{T} T^{T}, \quad M_{3,8}=-N g_{i j}^{2} \Gamma_{2}^{T} T^{T},
\end{aligned}
$$




$$
\begin{aligned}
M_{3,12} & =-N g_{i j}^{3} \Gamma_{3}^{T} T^{T}+D^{T} T^{T}, M_{4,4}=-\left(1-\mu_{1}\right) e^{-2 \alpha \tau_{2}} Q_{5} \\
M_{5,5} & =-\Omega-Q_{5}, \quad M_{6,6}=-\Theta, \quad M_{7,7}=k S_{3}-\Delta \\
M_{8,8} & =-\left(1-\mu_{1}\right) e^{-2 \alpha \tau_{2}} Q_{4}-e^{-2 \alpha \tau_{2}} R_{3}-e^{-2 \alpha \tau_{2}} R_{3}, \\
M_{8,9} & =-M^{-2 \alpha \tau_{2}} R_{3}, \quad M_{8,10}=-e^{-2 \alpha \tau_{2}} R_{3}, \\
M_{9,9} & =-e^{-2 \alpha \tau_{1}}\left(Q_{2}+R_{1}\right)-e^{-2 \alpha \tau_{2}} R_{3}, \\
M_{10,10} & =-e^{-2 \alpha \tau_{2}}\left(R_{2}+R_{3}+Q_{3}\right), \\
M_{11,11} & =-\frac{\left(1-\mu_{2}\right)}{\mu_{2}} e^{-2 \alpha k} S_{2}, \quad M_{12,12}=-\frac{\left(1-\mu_{2}\right)}{\mu_{2}} e^{-2 \alpha k} S_{3}, \\
0 & =M_{1,11}=M_{2,4}=M_{2,5}=M_{2,6}=M_{2,7}=M_{2,8}=M_{2,9} \\
& =M_{2,10}=M_{2,11}=M_{2,12}=M_{3,5}=M_{3,7}=M_{3,9}=M_{3,10} \\
& =M_{3,11}=M_{4,5}=M_{4,6}=M_{4,7}=M_{4,8}=M_{4,9}=M_{4,10} \\
& =M_{4,11}=M_{4,12}=M_{5,6}=M_{5,7}=M_{5,8}=M_{5,9}=M_{5,10} \\
& =M_{5,11}=M_{5,12}=M_{6,7}=M_{6,8}=M_{6,9}=M_{6,10}=M_{6,11} \\
& =M_{6,12}=M_{7,8}=M_{7,9}=M_{7,10}=M_{7,11}=M_{7,12}=M_{8,11} \\
& =M_{8,12}=M_{9,10}=M_{9,11}=M_{9,12}=M_{10,11}=M_{10,12} \\
& =M_{11,12} .
\end{aligned}
$$

Moreover, the feedback control is $K=-P^{-1} Y$ and the solution $\|x(t, \phi)\|$ of the system satisfies $\|x(t, \phi)\| \leq \sqrt{\frac{\lambda_{1}}{\lambda_{2}}} e^{-\alpha t}\|\phi\|, \forall t \geq 0$.

Proof. For the system (5), the following lemma provides a change estimation of Lyapunov-Krasovskii functional candidate:

$$
V(x(t))=\sum_{l=1}^{6} V_{l}(x(t))
$$

where

$$
\begin{aligned}
V_{1}(x(t)= & x^{T}(t)(U \otimes P) x(t) \\
V_{2}(x(t)= & \int_{t-\sigma}^{t} e^{2 \alpha(s-t)} x^{T}(s)\left(U \otimes Q_{1}\right) x(s) d s \\
& +\sum_{l=1}^{2} \int_{t-\tau_{l}}^{t} e^{2 \alpha(s-t)} x^{T}(s)\left(U \otimes Q_{l+1}\right) x(s) d s \\
& +\int_{t-\tau(t)}^{t} e^{2 \alpha(s-t)} x^{T}(s)\left(U \otimes Q_{4}\right) x(s) d s
\end{aligned}
$$




$$
\begin{aligned}
& +\int_{t-\tau(t)}^{t} e^{2 \alpha(s-t)} g\left(x^{T}(s)\right)\left(U \otimes Q_{5}\right) g(x(s)) d s, \\
V_{3}(x(t)= & \tau_{1} \int_{-\tau_{1}}^{0} \int_{t+s}^{t} e^{2 \alpha(\theta-t)} \dot{x}^{T}(\theta)\left(U \otimes R_{1}\right) \dot{x}(\theta) d \theta d s \\
& +\tau_{2} \int_{-\tau_{2}}^{0} \int_{t+s}^{t} e^{2 \alpha(\theta-t)} \dot{x}^{T}(\theta)\left(U \otimes R_{2}\right) \dot{x}(\theta) d \theta d s, \\
V_{4}(x(t)= & \eta \int_{-\tau_{2}}^{-\tau_{1}} \int_{t+s}^{t} e^{2 \alpha(\theta-t)} \dot{x}^{T}(\theta)\left(U \otimes R_{3}\right) \dot{x}(\theta) d \theta d s, \\
V_{5}(x(t)= & \sigma \int_{-\sigma}^{0} \int_{t+s}^{t} e^{2 \alpha(\theta-t)} \dot{x}^{T}(\theta)\left(U \otimes S_{1}\right) \dot{x}(\theta) d \theta d s, \\
V_{6}(x(t)= & \int_{-k(t)}^{0} \int_{t+s}^{t} e^{2 \alpha(\theta-t)} x^{T}(\theta)\left(U \otimes S_{2}\right) x(\theta) d \theta d s \\
& +\int_{-k(t)}^{0} \int_{t+s}^{t} e^{2 \alpha(\theta-t)} h^{T}(x(\theta))\left(U \otimes S_{3}\right) h(x(\theta)) d \theta d s .
\end{aligned}
$$

It easy to check that

$$
\lambda_{1}\|x(t)\|^{2} \leq V(x(t)) \leq \lambda_{2}\|x(t)\|^{2}, \quad \forall t \geq 0
$$

Calculating the time derivative of $V(x(t))$ along the trajectories of (5), noting that the fact that if $X$ is a matrix with zero column sums, then $U X=$ $N X$, we obtain:

$$
\begin{gathered}
\dot{V}_{1}=2 x^{T}(t)(U \otimes P) \dot{x}(t) \\
\dot{V}_{1}=2 \sum_{1 \leq i<j \leq N}^{N}\left(x_{i}(t)-x_{j}(t)\right)^{T}\left[\left(-N g_{i j}^{1} P \Gamma_{1}+P E K\right)\left(x_{i}(t)-x_{j}(t)\right)\right. \\
-P C\left(x_{i}(t-\sigma)-x_{j}(t-\sigma)\right)+P A\left(f\left(x_{i}(t)\right)-f\left(x_{j}(t)\right)\right) \\
+P B\left(g\left(x_{i}(t-\tau(t))\right)-g\left(x_{j}(t-\tau(t))\right)\right) \\
+\left(P D-N g_{i j}^{3} P \Gamma_{3}\right) \int_{t-k(t)}^{t} k(t-s)\left(h\left(x_{i}(s)\right)-h\left(x_{j}(s)\right)\right) d s \\
\left.-N g_{i j}^{2} P \Gamma_{2}\left(x_{i}(t-\tau(t))-x_{j}(t-\tau(t))\right)\right] .
\end{gathered}
$$

From Lemma 4, we have

$$
-\sum_{1 \leq i<j \leq N}^{N} 2\left(x_{i}(t)-x_{j}(t)\right)^{T} P E P^{-1} Y\left(x_{i}(t)-x_{j}(t)\right)
$$




$$
\leq \sum_{1 \leq i<j \leq N}^{N}\left(x_{i}(t)-x_{j}(t)\right)^{T}\left(P E P^{-1} E^{T} P+Y^{T} P^{-1} Y\right)\left(x_{i}(t)-x_{j}(t)\right)
$$

Next, the derivatives of $V_{l}(x(t)), l=2,3, \ldots, 6$, we have

$$
\begin{aligned}
\dot{V}_{2} \leq & \sum_{1 \leq i<j \leq N}^{N}\left\{\left(x_{i}(t)-x_{j}(t)\right)^{T}\left(Q_{1}+Q_{2}+Q_{3}+Q_{4}\right)\left(x_{i}(t)-x_{j}(t)\right)\right. \\
& -e^{-2 \alpha \sigma}\left(x_{i}(t-\sigma)-x_{j}(t-\sigma)\right)^{T} Q_{1}\left(x_{i}(t-\sigma)-x_{j}(t-\sigma)\right) \\
& -e^{-2 \alpha \tau_{1}}\left(x_{i}\left(t-\tau_{1}\right)-x_{j}\left(t-\tau_{1}\right)\right)^{T} Q_{2}\left(x_{i}\left(t-\tau_{1}\right)-x_{j}\left(t-\tau_{1}\right)\right) \\
& -e^{-2 \alpha \tau_{2}}\left(x_{i}\left(t-\tau_{2}\right)-x_{j}\left(t-\tau_{2}\right)\right)^{T} Q_{3}\left(x_{i}\left(t-\tau_{2}\right)-x_{j}\left(t-\tau_{2}\right)\right) \\
& +\left(g\left(x_{i}(t)\right)-g\left(x_{j}(t)\right)\right)^{T} Q_{5}\left(g\left(x_{i}(t)\right)-g\left(x_{j}(t)\right)\right)-\left(1-\mu_{1}\right) \\
& \times e^{-2 \alpha \tau_{2}}\left[( x _ { i } ( t - \tau ( t ) ) - x _ { j } ( t - \tau ( t ) ) ) ^ { T } Q _ { 4 } \left(x_{i}(t-\tau(t))\right.\right. \\
& -x_{j}(t-\tau(t))+\left(g\left(x_{i}(t-\tau(t))\right)-g\left(x_{j}(t-\tau(t))\right)\right)^{T} Q_{5} \\
& \left.\times\left(g\left(x_{i}(t-\tau(t))\right)-g\left(x_{j}(t-\tau(t))\right)\right]\right\}-2 \alpha V_{2} \cdot \\
\dot{V}_{3} \leq & \sum^{N}\left\{\left(\dot{x}_{i}(t)-\dot{x}_{j}(t)\right)^{T}\left(\tau_{1}^{2} R_{1}+\tau_{2}^{2} R_{2}\right)\left(\dot{x}_{i}(t)-\dot{x}_{j}(t)\right)\right. \\
& -i \leq j \leq N \\
& -e^{-2 \alpha \tau_{1}}\left[\left(x_{i}(t)-x_{j}(t)\right)-\left(x_{i}\left(t-\tau_{1}\right)-x_{j}\left(t-\tau_{1}\right)\right)\right]^{T} R_{1} \\
& \times\left[\left(x_{i}(t)-x_{j}(t)\right)-\left(x_{i}\left(t-\tau_{1}\right)-x_{j}\left(t-\tau_{1}\right)\right)\right] \\
& -e^{-2 \alpha \tau_{2}}\left[\left(x_{i}(t)-x_{j}(t)\right)-\left(x_{i}\left(t-\tau_{2}\right)-x_{j}\left(t-\tau_{2}\right)\right)\right]^{T} R_{2} \\
& \left.\times\left[\left(x_{i}(t)-x_{j}(t)\right)-\left(x_{i}\left(t-\tau_{2}\right)-x_{j}\left(t-\tau_{2}\right)\right)\right]\right\}-2 \alpha V_{3} . \\
\dot{V}_{4}= & \eta^{2} \dot{x}^{T}(t)\left(U \otimes R_{3}\right) \dot{x}(t)-\eta e^{-2 \alpha \tau_{2}} \int_{t-\tau_{1}} \dot{x}^{T}(s)\left(U R_{3}\right) \dot{x}(s) d s \\
& -2 \alpha V_{4},
\end{aligned}
$$

let $\eta=\left(\tau_{2}-\tau(t)\right)+\left(\tau(t)-\tau_{1}\right)$, we have

$$
\begin{aligned}
-\eta & \int_{t-\tau_{2}}^{t-\tau_{1}} \dot{x}^{T}(s)\left(U \otimes R_{3}\right) \dot{x}(s) d s \\
= & -\left(\tau_{2}-\tau(t)\right) \int_{t-\tau_{2}}^{t-\tau(t)} \dot{x}^{T}(s)\left(U \otimes R_{3}\right) \dot{x}(s) d s \\
& -\left(\tau(t)-\tau_{1}\right) \int_{t-\tau_{2}}^{t-\tau(t)} \dot{x}^{T}(s)\left(U \otimes R_{3}\right) \dot{x}(s) d s \\
& -\left(\tau_{2}-\tau(t)\right) \int_{t-\tau(t)}^{t-\tau_{1}} \dot{x}^{T}(s)\left(U \otimes R_{3}\right) \dot{x}(s) d s
\end{aligned}
$$




$$
-\left(\tau(t)-\tau_{1}\right) \int_{t-\tau(t)}^{t-\tau_{1}} \dot{x}^{T}(s)\left(U \otimes R_{3}\right) \dot{x}(s) d s .
$$

Let $\beta=\frac{\tau_{2}-\tau(t)}{\tau_{2}-\tau_{1}} \leq 1$ and Lemma 3 , we have

$$
\begin{aligned}
& -\left(\tau_{2}-\tau(t)\right) \int_{t-\tau_{2}}^{t-\tau(t)} \dot{x}^{T}(s)\left(U \otimes R_{3}\right) \dot{x}(s) d s \\
& \leq\left[\begin{array}{c}
x(t-\tau(t)) \\
x\left(t-\tau_{2}\right)
\end{array}\right]^{T}\left[\begin{array}{cc}
-\left(U \otimes R_{3}\right) & \left(U \otimes R_{3}\right) \\
* & -\left(U \otimes R_{3}\right)
\end{array}\right]\left[\begin{array}{c}
x(t-\tau(t)) \\
x\left(t-\tau_{2}\right)
\end{array}\right], \\
& -\left(\tau(t)-\tau_{1}\right) \int_{t-\tau(t)}^{t-\tau_{1}} \dot{x}^{T}(s)\left(U \otimes R_{3}\right) \dot{x}(s) d s \\
& \leq\left[\begin{array}{cc}
x(t-\tau(t)) \\
x\left(t-\tau_{1}\right)
\end{array}\right]^{T}\left[\begin{array}{cc}
-\left(U \otimes R_{3}\right) & \left(U \otimes R_{3}\right) \\
* & -\left(U \otimes R_{3}\right)
\end{array}\right]\left[\begin{array}{c}
x(t-\tau(t)) \\
x\left(t-\tau_{1}\right)
\end{array}\right], \\
& -\left(\tau_{2}-\tau(t)\right) \int_{t-\tau(t)}^{t-\tau_{1}} \dot{x}^{T}(s)\left(U \otimes R_{3}\right) \dot{x}(s) d s \quad \leq \beta \\
& \times\left[\begin{array}{cc}
x(t-\tau(t)) \\
x\left(t-\tau_{1}\right)
\end{array}\right]^{T}\left[\begin{array}{cc}
-\left(U \otimes R_{3}\right) & \left(U \otimes R_{3}\right) \\
* & -\left(U \otimes R_{3}\right)
\end{array}\right]\left[\begin{array}{c}
x(t-\tau(t)) \\
x\left(t-\tau_{1}\right)
\end{array}\right], \\
& \left.-\left(\tau(t)-\tau_{1}\right) \int_{t-\tau_{2}}^{t-\tau(t)} \begin{array}{cc}
\dot{x}^{T}(s)\left(U \otimes R_{3}\right) \dot{x}(s) d s & \leq(1-\beta) \\
-\left(U \otimes R_{3}\right) & \left(U \otimes R_{3}\right) \\
* & -\left(U \otimes R_{3}\right)
\end{array}\right]\left[\begin{array}{c}
x(t-\tau(t)) \\
x\left(t-\tau_{2}\right)
\end{array}\right] .
\end{aligned}
$$

Together with the terms in (16)-(21), we can estimate $\dot{V}_{4}(t)$ as

$$
\begin{aligned}
\dot{V}_{4} \leq & \sum_{1 \leq i<j \leq N}^{N}\left\{\left(\dot{x}_{i}(t)-\dot{x}_{j}(t)\right)^{T} \eta^{2} R_{3}\left(\dot{x}_{i}(t)-\dot{x}_{j}(t)\right)-e^{-2 \alpha \tau_{2}}\right. \\
& \times\left[\left(x_{i}(t-\tau(t))-x_{j}(t-\tau(t))\right)-\left(x_{i}\left(t-\tau_{2}\right)-x_{j}\left(t-\tau_{2}\right)\right)\right]^{T} \\
& R_{3}\left[\left(x_{i}(t-\tau(t))-x_{j}(t-\tau(t))\right)-\left(x_{i}\left(t-\tau_{2}\right)-x_{j}\left(t-\tau_{2}\right)\right)\right] \\
& -e^{-2 \alpha \tau_{2}}\left[\left(x_{i}\left(t-\tau_{1}\right)-x_{j}\left(t-\tau_{1}\right)\right)-\left(x_{i}(t-\tau(t))\right.\right. \\
& \left.\left.-x_{j}(t-\tau(t))\right)\right]^{T} R_{3}\left[\left(x_{i}\left(t-\tau_{1}\right)-x_{j}\left(t-\tau_{1}\right)\right)-\left(x_{i}(t-\tau(t))\right.\right. \\
& \left.\left.-x_{j}(t-\tau(t))\right)\right]-\beta e^{-2 \alpha \tau_{2}}\left[\left(x_{i}\left(t-\tau_{1}\right)-x_{j}\left(t-\tau_{1}\right)\right)\right. \\
& \left.-\left(x_{i}(t-\tau(t))-x_{j}(t-\tau(t))\right)\right]^{T} R_{3}\left[\left(x_{i}\left(t-\tau_{1}\right)-x_{j}\left(t-\tau_{1}\right)\right)\right. \\
& \left.-\left(x_{i}(t-\tau(t))-x_{j}(t-\tau(t))\right)\right]-(1-\beta) e^{-2 \alpha \tau_{2}}\left[\left(x_{i}(t-\tau(t))\right.\right. \\
& \left.\left.-x_{j}(t-\tau(t))\right)-\left(x_{i}\left(t-\tau_{2}\right)-x_{j}\left(t-\tau_{2}\right)\right)\right]^{T} R_{3}\left[\left(x_{i}(t-\tau(t))\right.\right.
\end{aligned}
$$




$$
\begin{aligned}
& \left.\left.\left.-x_{j}(t-\tau(t))\right)-\left(x_{i}\left(t-\tau_{2}\right)-x_{j}\left(t-\tau_{2}\right)\right)\right]\right\}-2 \alpha V_{4} . \\
\dot{V}_{5} \leq & \sum_{1 \leq i<j \leq N}^{N}\left\{\left(\dot{x}_{i}(t)-\dot{x}_{j}(t)\right)^{T} \sigma^{2} S_{1}\left(\dot{x}_{i}(t)-\dot{x}_{j}(t)\right)\right. \\
& -e^{-2 \alpha \sigma}\left[\left(x_{i}(t)-x_{j}(t)\right)-\left(x_{i}(t-\sigma)-x_{j}(t-\sigma)\right)\right]^{T} S_{1} \\
& \left.\times\left[\left(x_{i}(t)-x_{j}(t)\right)-\left(x_{i}(t-\sigma)-x_{j}(t-\sigma)\right)\right]\right\}-2 \alpha V_{5} . \\
\dot{V}_{6} \leq & \sum_{1 \leq i<j \leq N}^{N}\left\{\left(x_{i}(t)-x_{j}(t)\right)^{T} k S_{2}\left(x_{i}(t)-x_{j}(t)\right)\right. \\
& +\left[h\left(x_{i}(t)\right)-h\left(x_{j}(t)\right)\right]^{T} k S_{3}\left[h\left(x_{i}(t)\right)-h\left(x_{j}(t)\right)\right] \\
& -\frac{\left(1-\mu_{2}\right)}{\mu_{2}} e^{-2 \alpha k}\left(\int_{t-k(t)}^{t} k(t-s)\left[x_{i}(s)-x_{j}(s)\right] d s\right)^{T} \\
& \times S_{2}\left(\int_{t-k(t)}^{t} k(t-s)\left[x_{i}(s)-x_{j}(s)\right] d s\right) \\
& -\frac{\left(1-\mu_{2}\right)}{\mu_{2}} e^{-2 \alpha k}\left(\int_{t-k(t)}^{t} k(t-s)\left[h\left(x_{i}(s)\right)-h\left(x_{j}(s)\right)\right] d s\right)^{T} \\
& \left.\times S_{3}\left(\int_{t-k(t)}^{t} k(t-s)\left[h\left(x_{i}(s)\right)-h\left(x_{j}(s)\right)\right] d s\right)\right\}-2 \alpha V_{6} .
\end{aligned}
$$

By assumption 2, for any diagonal matrix $\Omega>0, \Theta>0, \Delta>0$ with appropriate dimensions, it follows that:

$$
\begin{aligned}
& {\left[\begin{array}{c}
x_{i}(t)-x_{j}(t) \\
f\left(x_{i}(t)\right)-f\left(x_{j}(t)\right)
\end{array}\right]^{T}\left[\begin{array}{cc}
-\Omega F_{1} & \Omega F_{2} \\
\Omega F_{2} & -\Omega
\end{array}\right]\left[\begin{array}{c}
x_{i}(t)-x_{j}(t) \\
f\left(x_{i}(t)\right)-f\left(x_{j}(t)\right)
\end{array}\right] } \\
+ & {\left[\begin{array}{c}
x_{i}(t)-x_{j}(t) \\
g\left(x_{i}(t)\right)-g\left(x_{j}(t)\right)
\end{array}\right]^{T}\left[\begin{array}{cc}
-\Theta J_{1} & \Theta J_{2} \\
\Theta J_{2} & -\Theta
\end{array}\right]\left[\begin{array}{c}
x_{i}(t)-x_{j}(t) \\
g\left(x_{i}(t)\right)-g\left(x_{j}(t)\right)
\end{array}\right] } \\
+ & {\left[\begin{array}{c}
x_{i}(t)-x_{j}(t) \\
h\left(x_{i}(t)\right)-h\left(x_{j}(t)\right)
\end{array}\right]^{T}\left[\begin{array}{cc}
-\Delta L_{1} & \Delta L_{2} \\
\Delta L_{2} & -\Delta
\end{array}\right]\left[\begin{array}{c}
x_{i}(t)-x_{j}(t) \\
h\left(x_{i}(t)\right)-h\left(x_{j}(t)\right)
\end{array}\right] } \\
& \geq 0 .
\end{aligned}
$$

On the other hand, it is easy to see from the formulation of (5) that the following equations also holds for any matrices $T$,

$$
\begin{aligned}
0= & 2 \sum_{\substack{1 \leq i<j \leq N \\
N}}^{N}\left(\dot{x}_{i}(t)-\dot{x}_{j}(t)\right)^{T}\left[\left(-N g_{i j}^{1} T \Gamma_{1}+T E K\right)\left(x_{i}(t)-x_{j}(t)\right)\right. \\
& -T C\left(x_{i}(t-\sigma)-x_{j}(t-\sigma)\right)+T A\left(f\left(x_{i}(t)\right)-f\left(x_{j}(t)\right)\right)
\end{aligned}
$$




$$
\begin{aligned}
& +T B\left(g\left(x_{i}(t-\tau(t))\right)-g\left(x_{j}(t-\tau(t))\right)\right)+\left(T D-N g_{i j}^{3} T \Gamma_{3}\right) \\
& \times \int_{t-k(t)}^{t} k(t-s)\left(h\left(x_{i}(s)\right)-h\left(x_{j}(s)\right)\right) d s-N g_{i j}^{2} T \Gamma_{2} \\
& \left.\times\left(x_{i}(t-\tau(t))-x_{j}(t-\tau(t))\right)-T\left(\dot{x}_{i}(t)-\dot{x}_{j}(t)\right)\right] .
\end{aligned}
$$

By Lemma 4, we have

$$
\begin{aligned}
& -\sum_{1 \leq i<j \leq N}^{N} 2\left(\dot{x}_{i}(t)-\dot{x}_{j}(t)\right)^{T} T E P^{-1} Y\left(x_{i}(t)-x_{j}(t)\right) \\
& \leq \sum_{\substack{1 \leq i<j \leq N \\
N}}^{N}\left[\left(\dot{x}_{i}(t)-\dot{x}_{j}(t)\right)^{T} T E P^{-1} E^{T} T^{T}\left(\dot{x}_{i}(t)-\dot{x}_{j}(t)\right)\right. \\
& \left.\quad+\left(x_{i}(t)-x_{j}(t)\right)^{T} Y^{T} P^{-1} Y\left(x_{i}(t)-x_{j}(t)\right)\right] .
\end{aligned}
$$

By setting $\zeta_{i j}=\left[\left(x_{i}(t)-x_{j}(t)\right)^{T},\left(x_{i}(t-\sigma)-x_{j}(t-\sigma)\right)^{T},\left(\dot{x}_{i}(t)-\dot{x}_{j}(t)\right)^{T},\left(g\left(x_{i}(t-\right.\right.\right.$ $\left.\tau(t)))-g\left(x_{j}(t-\tau(t))\right)\right)^{T},\left(g\left(x_{i}(t)\right)-g\left(x_{j}(t)\right)\right)^{T},\left(f\left(x_{i}(t)\right)-f\left(x_{j}(t)\right)\right)^{T},\left(h\left(x_{i}(t)\right)-\right.$ $\left.h\left(x_{j}(t)\right)\right)^{T},\left(x_{i}(t-\tau(t))-x_{j}(t-\tau(t))\right)^{T},\left(x_{i}\left(t-\tau_{1}\right)-x_{j}\left(t-\tau_{1}\right)\right)^{T},\left(x_{i}\left(t-\tau_{2}\right)-\right.$ $\left.\left.x_{j}\left(t-\tau_{2}\right)\right)^{T}, \int_{-\infty}^{t} k(t-s)\left(x_{i}(s)-x_{j}(s)\right)^{T} d s, \int_{-\infty}^{t} k(t-s)\left(h\left(x_{i}(s)\right)-h\left(x_{j}(s)\right)\right)^{T} d s\right]$ it can be obtained from (12)-(26) that

$$
\begin{aligned}
\dot{V}(x(t)) \leq & \sum_{1 \leq i<j \leq N}^{N}\left\{\zeta_{i j}^{T}(t)\left[(1-\beta) \Sigma_{1 i j}+\beta \Sigma_{2 i j}\right] \zeta_{i j}(t)\right. \\
& +\left(x_{i}(t)-x_{j}(t)\right)^{T} \Xi_{1}\left(x_{i}(t)-x_{j}(t)\right)+\left(\dot{x}_{i}(t)-\dot{x}_{j}(t)\right)^{T} \Xi_{2} \\
& \left.\times\left(\dot{x}_{i}(t)-\dot{x}_{j}(t)\right)\right\}-2 \alpha V(x(t)),
\end{aligned}
$$

where $\Sigma_{1 i j}$ and $\Sigma_{2 i j}$ are defined in (6), (7), respectively, and

$$
\begin{aligned}
& \Xi_{1}=-0.1 e^{-2 \alpha \tau_{1}} R_{1}-0.1 e^{-2 \alpha \tau_{2}} R_{2}+P E P^{-1} E^{T} P+2 Y^{T} P^{-1} Y, \\
& \Xi_{2}=-T+T E P^{-1} E^{T} T^{T} .
\end{aligned}
$$

Since $0 \leq \beta \leq 1,(1-\beta) \Sigma_{1 i j}+\beta \Sigma_{2 i j}$ is a convex combination of $\Sigma_{1 i j}$ and $\Sigma_{2 i j}$. Therefore, $(1-\beta) \Sigma_{1 i j}+\beta \Sigma_{2 i j}<0$ is equivalent to $\Sigma_{1 i j}<0$ and $\Sigma_{2 i j}<0$. Applying Shur complement lemma, the inequalities $\Xi_{1}$ and $\Xi_{2}$ are equivalent to $\Sigma_{3}<0$ and $\Sigma_{4}<0$. Thus, it follows from (6)-(9) and (27) such that

$$
\dot{V}(x(t))+2 \alpha V(x(t)) \leq 0, \quad \forall t \geq 0 .
$$

Integrating both sides of (28) from 0 to $t$, we obtain

$$
V(x(t)) \leq e^{-2 \alpha t} V(\phi), \quad \forall t \geq 0 .
$$


Furthermore, taking condition (11) into account, we have

$$
\lambda_{1}\|x(t, \phi)\|^{2} \leq V(x(t)) \leq e^{-2 \alpha t} V(\phi) \leq \lambda_{2} e^{-2 \alpha t}\|\phi\|^{2}, \quad \forall t \geq 0 .
$$

then

$$
\|x(t)\| \leq \sqrt{\frac{\lambda_{2}}{\lambda_{1}}} e^{-\alpha t}\|\phi\|, \quad \forall t \geq 0 .
$$

This completes the proof.

Theorem 3. Given $0<\alpha<\varepsilon$, and suppose that assumption 1-2 hold, the system (5) is exponentially synchronized under the controller (4) if there exist symmetric positive definite matrices, $P, Q_{k}, R_{l}, S_{l}, Y(k=1,2, \ldots, 5, l=1,2,3)$ and positive diagonal matrix $\Omega, \Theta, \Delta$ with appropriate dimensions, such that the following linear matrix inequalities (LMIs) hold for all $1=i<j=N$ :

$$
\begin{aligned}
& \Sigma_{5 i j}=\mathcal{Z}_{i j}-\left[\begin{array}{llllllllllll}
0 & 0 & 0 & 0 & 0 & 0 & 0 & I & 0 & -I & 0 & 0
\end{array}\right]^{T} e^{-2 \alpha \tau_{2}} \\
& \times R_{3}\left[\begin{array}{llllllllllll}
0 & 0 & 0 & 0 & 0 & 0 & 0 & I & 0 & -I & 0 & 0
\end{array}\right]<0 \text {, } \\
& \Sigma_{6 i j}=\mathcal{Z}_{i j}-\left[\begin{array}{llllllllllll}
0 & 0 & 0 & 0 & 0 & 0 & 0 & -I & I & 0 & 0 & 0
\end{array}\right]^{T} e^{-2 \alpha \tau_{2}} \\
& \times R_{3}\left[\begin{array}{llllllllllll}
0 & 0 & 0 & 0 & 0 & 0 & 0 & -I & I & 0 & 0 & 0
\end{array}\right]<0, \\
& \Sigma_{7}=\left[\begin{array}{ccc}
-0.1 e^{-2 \alpha \tau_{1}} R_{1}-0.1 e^{-2 \alpha \tau_{2}} R_{2} & 2 Y^{T} & P E \\
* & -2 P & 0 \\
* & * & -P
\end{array}\right]<0, \\
& \Sigma_{8}=\left[\begin{array}{cc}
-T & T E \\
* & -P
\end{array}\right]<0, \\
& \Sigma_{9 i j}=\widehat{\mathcal{Z}}_{i j}-\left[\begin{array}{llllllllllll}
0 & 0 & 0 & 0 & 0 & 0 & 0 & I & 0 & -I & 0 & 0
\end{array}\right]^{T} e^{-2 \alpha \tau_{2}} \\
& \times R_{3}\left[\begin{array}{lllllllllllll}
0 & 0 & 0 & 0 & 0 & 0 & 0 & I & 0 & -I & 0 & 0
\end{array}\right]<0, \\
& \Sigma_{10 i j}=\widehat{\mathcal{Z}}_{i j}-\left[\begin{array}{llllllllllll}
0 & 0 & 0 & 0 & 0 & 0 & 0 & -I & I & 0 & 0 & 0
\end{array}\right]^{T} e^{-2 \alpha \tau_{2}} \\
& \times R_{3}\left[\begin{array}{llllllllllll}
0 & 0 & 0 & 0 & 0 & 0 & 0 & -I & I & 0 & 0 & 0
\end{array}\right]<0,
\end{aligned}
$$

and

$$
-\alpha \delta+(\varepsilon-\alpha)(\omega-\delta)<0
$$

where

$$
\mathcal{Z}_{i j}=\left[\begin{array}{ccccc}
Z_{1,1} & Z_{1,2} & Z_{1,3} & \cdots & Z_{1,12} \\
* & Z_{2,2} & Z_{2,3} & \cdots & Z_{2,12} \\
* & * & Z_{3,3} & \cdots & Z_{3,12} \\
\vdots & \vdots & \vdots & \ddots & \vdots \\
* & * & * & \cdots & Z_{12,12}
\end{array}\right]
$$




$$
\begin{aligned}
& \widehat{\mathcal{Z}}_{i j}=\left[\begin{array}{ccccc}
\widehat{Z}_{1,1} & Z_{1,2} & Z_{1,3} & \cdots & Z_{1,12} \\
* & Z_{2,2} & Z_{2,3} & \cdots & Z_{2,12} \\
* & * & \widehat{Z}_{3,3} & \cdots & Z_{3,12} \\
\vdots & \vdots & \vdots & \ddots & \vdots \\
* & * & * & \cdots & Z_{12,12}
\end{array}\right] \\
& Z_{1,1}=-N g_{i j}^{1} P \Gamma_{1}-N g_{i j}^{1} \Gamma_{1}^{T} P+\sum_{r=1}^{4} Q_{r}-0.9 e^{-2 \alpha \tau_{1}} R_{1} \\
& -0.9 e^{-2 \alpha \tau_{2}} R_{2}-e^{-2 \alpha \sigma} S_{1}+k S_{2}-\Omega F_{1}-\Theta J_{1}-\Delta L_{1}, \\
& Z_{1,2}=-e^{-2 \alpha \sigma} S_{1}+C^{T} P, \quad Z_{1,3}=-N g_{i j}^{1} T \Gamma_{1}, \\
& Z_{1,4}=B^{T} P, \quad Z_{1,5}=\Omega F_{2}, \\
& Z_{1,6}=\Theta J_{2}+A^{T} P, \quad Z_{1,7}=\Delta L_{2}, \\
& Z_{1,8}=-N g_{i j}^{2} \Gamma_{2}^{T} P, \quad Z_{1,9}=e^{-2 \alpha \tau_{1}} R_{1}, \\
& Z_{1,10}=e^{-2 \alpha \tau_{2}} R_{2}, \quad Z_{1,12}=-N g_{i j}^{3} \Gamma_{3}^{T} P+D^{T} P, \\
& Z_{2,2}=-e^{-2 \alpha \sigma}\left(Q_{1}+S_{1}\right), \quad Z_{2,3}=-T C, \\
& Z_{3,3}=\tau_{1}^{2} R_{1}+\tau_{2}^{2} R_{2}+\eta^{2} R_{3}+\sigma^{2} S_{1}-T, \\
& Z_{3,4}=B^{T} T, \quad Z_{3,6}=A^{T} T^{T}, \quad Z_{3,8}=-N g_{i j}^{2} \Gamma_{2}^{T} T^{T}, \\
& Z_{3,12}=-N g_{i j}^{3} \Gamma_{3}^{T} T^{T}+D^{T} T^{T}, Z_{4,4}=-\left(1-\mu_{1}\right) e^{-2 \alpha \tau_{2}} Q_{5}, \\
& Z_{5,5}=-\Omega-Q_{5}, \quad Z_{6,6}=-\Theta, \quad Z_{7,7}=k S_{3}-\Delta, \\
& Z_{8,8}=-\left(1-\mu_{1}\right) e^{-2 \alpha \tau_{2}} Q_{4}-e^{-2 \alpha \tau_{2}} R_{3}-e^{-2 \alpha \tau_{2}} R_{3}, \\
& Z_{8,9}=-e^{-2 \alpha \tau_{2}} R_{3}, \quad Z_{8,10}=-e^{-2 \alpha \tau_{2}} R_{3}, \\
& Z_{9,9}=-e^{-2 \alpha \tau_{1}}\left(Q_{2}+R_{1}\right)-e^{-2 \alpha \tau_{2}} R_{3}, \\
& Z_{10,10}=-e^{-2 \alpha \tau_{2}}\left(R_{2}+R_{3}+Q_{3}\right), \\
& Z_{11,11}=-\frac{\left(1-\mu_{2}\right)}{\mu_{2}} e^{-2 \alpha k} S_{2}, \quad Z_{12,12}=-\frac{\left(1-\mu_{2}\right)}{\mu_{2}} e^{-2 \alpha k} S_{3}, \\
& 0=Z_{1,11}=Z_{2,4}=Z_{2,5}=Z_{2,6}=Z_{2,7}=Z_{2,8}=Z_{2,9} \\
& =Z_{2,10}=Z_{2,11}=Z_{2,12}=Z_{3,5}=Z_{3,7}=Z_{3,9}=Z_{3,10} \\
& =Z_{3,11}=Z_{4,5}=Z_{4,6}=Z_{4,7}=Z_{4,8}=Z_{4,9}=Z_{4,10} \\
& =Z_{4,11}=Z_{4,12}=Z_{5,6}=Z_{5,7}=Z_{5,8}=Z_{5,9}=Z_{5,10} \\
& =Z_{5,11}=Z_{5,12}=Z_{6,7}=Z_{6,8}=Z_{6,9}=Z_{6,10}=Z_{6,11} \\
& =Z_{6,12}=Z_{7,8}=Z_{7,9}=Z_{7,10}=Z_{7,11}=Z_{7,12}=Z_{8,11} \\
& =Z_{8,12}=Z_{9,10}=Z_{9,11}=Z_{9,12}=Z_{10,11}=Z_{10,12}=Z_{11,12} \text {, }
\end{aligned}
$$




$$
\begin{aligned}
\widehat{Z}_{1,1}= & -N g_{i j}^{1} P \Gamma_{1}-N g_{i j}^{1} \Gamma_{1}^{T} P+\sum_{r=1}^{4} Q_{r}-e^{-2 \alpha \tau_{1}} R_{1}-e^{-2 \alpha \tau_{2}} R_{2} \\
& -e^{-2 \alpha \sigma} S_{1}+k S_{2}-\Omega F_{1}-\Theta J_{1}-\Delta L_{1}-2 \varepsilon P, \\
\widehat{Z}_{3,3}= & \tau_{1}^{2} R_{1}+\tau_{2}^{2} R_{2}+\eta^{2} R_{3}+\sigma^{2} S_{1}-T-T^{T} .
\end{aligned}
$$

Moreover, the memoryless control is $K=-P^{-1} Y$.

Proof. Case I: for $n \omega \leq t \leq n \omega+\delta$, we choose Lyapunov-Krasovskii functional as in (10), we may proof this case by using a similar argument as in the proof of Theorem 2. We have the following

$$
\begin{aligned}
\dot{V}(x(t)) \leq & \sum_{1 \leq i<j \leq N}^{N}\left\{\zeta_{i j}^{T}(t)\left[(1-\beta) \Sigma_{5 i j}+\beta \Sigma_{6 i j}\right] \zeta_{i j}(t)\right. \\
& +\left(x_{i}(t)-x_{j}(t)\right)^{T} \Upsilon_{1}\left(x_{i}(t)-x_{j}(t)\right)+\left(\dot{x}_{i}(t)-\dot{x}_{j}(t)\right)^{T} \\
& \left.\times \Upsilon_{2}\left(\dot{x}_{i}(t)-\dot{x}_{j}(t)\right)\right\}-2 \alpha V(x(t)),
\end{aligned}
$$

where $\Sigma_{5 i j}$ and $\Sigma_{6 i j}$ are defined in (31), (32), respectively, and

$$
\begin{aligned}
& \Upsilon_{1}=-0.1 e^{-2 \alpha \tau_{1}} R_{1}-0.1 e^{-2 \alpha \tau_{2}} R_{2}+P E P^{-1} E^{T} P+2 Y^{T} P^{-1} Y, \\
& \Upsilon_{2}=-T+T E P^{-1} E^{T} T^{T} .
\end{aligned}
$$

By $(1-\beta) \Sigma_{5 i j}+\beta \Sigma_{6 i j}<0$ is equivalent to $\Sigma_{5 i j}<0$ and $\Sigma_{6 i j}<0$. Applying Shur complement lemma, the inequalities $\Upsilon_{1}$ and $\Upsilon_{2}$ are equivalent to $\Sigma_{7}<0$ and $\Sigma_{8}<0$. Thus, it follows from (31)-(34) and (38), we obtain

$$
\dot{V}(x(t))+2 \alpha V(x(t)) \leq 0, \text { for } n \omega \leq t \leq n \omega+\delta .
$$

Thus, by the above differential inequality (39), we have

$$
V(x(t)) \leq V(x(n \omega)) e^{-2 \alpha(t-n \omega)}, \quad \text { for } \quad n \omega \leq t \leq n \omega+\delta .
$$

Case II: for $n \omega+\delta \leq t \leq(n+1) \omega$, we choose Lyapunov-Krasovskii functional having the following form :

$$
V(x(t))=\sum_{l=1}^{6} V_{l}(x(t)),
$$

where $V(x(t))$ are defined similar in (10). We are able to do similar estimation as we did in the Theorem 2 and add $2 \varepsilon V(x(t))-2 \varepsilon V_{1}(x(t))$. So, we obtain:

$$
\dot{V}(x(t))-2(\varepsilon-\alpha) V(x(t))
$$




$$
\leq \sum_{1 \leq i<j \leq N}^{N} \zeta_{i j}^{T}(t)\left[(1-\beta) \Sigma_{9 i j}+\beta \Sigma_{10 i j}\right] \zeta_{i j}(t),
$$

where $\Sigma_{9 i j}$ and $\Sigma_{10 i j}$ are defined in (35), (36), respectively.

By $(1-\beta) \Sigma_{9 i j}+\beta \Sigma_{10 i j}<0$ holds if and only if $\Sigma_{9 i j}<0$ and $\Sigma_{10 i j}<0$. Thus, it follows from (35)-(36) and (42), we obtain

$$
\dot{V}(x(t))-2(\varepsilon-\alpha) V(x(t)) \leq 0, \quad n \omega+\delta<t \leq(n+1) \omega .
$$

From the above differential inequality (43), we have

$$
V(x(t)) \leq V(x(n \omega+\delta)) e^{2(\varepsilon-\alpha)(t-n \omega-\delta)}, n \omega+\delta<t \leq(n+1) \omega .
$$

By (40) and (44), we have

$$
\begin{aligned}
V(x((n+1) \omega)) \leq & V(x(n \omega+\delta)) e^{2(\varepsilon-\alpha)(\omega-\delta)} \\
\leq & V(x(n \omega)) e^{-2 \alpha \delta} e^{2(\varepsilon-\alpha)(\omega-\delta)} \\
= & V(x(n \omega)) e^{-2 \alpha \delta+2(\varepsilon-\alpha)(\omega-\delta)} \\
\leq & V(x((n-1) \omega+\delta)) e^{2 \rho(\omega-\delta)} e^{-2 \alpha \delta+2(\varepsilon-\alpha)(\omega-\delta)} \\
\leq & V(x((n-1) \omega)) e^{-2 \alpha \delta+2(\varepsilon-\alpha)(\omega-\delta)} \\
& \times e^{-2 \alpha \delta+2(\varepsilon-\alpha)(\omega-\delta)} \\
= & V(((n-1) \omega)) e^{2(-2 \alpha \delta+2(\varepsilon-\alpha)(\omega-\delta))} \\
& \vdots \\
\leq & V(x(0)) e^{(-2 \alpha \delta+2(\varepsilon-\alpha)(\omega-\delta))(n+1)} .
\end{aligned}
$$

For any $t>0$, there is a $n_{0} \geq 0$, such that $n_{0} \omega \leq t \leq\left(n_{0}+1\right) \omega$.

Case 1. For $n_{0} \omega+\delta \leq t \leq\left(n_{0}+1\right) \omega$, using condition (37), we have

$$
\begin{aligned}
V(x(t)) & \leq V\left(x\left(n_{0} \omega+\delta\right)\right) e^{2(\varepsilon-\alpha)\left(t-\left(n_{0} \omega+\delta\right)\right)} \\
& \leq V\left(x\left(n_{0} \omega\right)\right) e^{-2 \alpha \delta} e^{2(\varepsilon-\alpha)\left(t-\left(n_{0} \omega+\delta\right)\right)} \\
& \leq V(x(0)) e^{(-2 \alpha \delta+2(\varepsilon-\alpha)(\omega-\delta)) n_{0}} e^{-2 \alpha \delta} e^{2(\varepsilon-\alpha)\left(t-\left(n_{0} \omega+\delta\right)\right)} \\
& \leq V(x(0)) e^{(-2 \alpha \delta+2(\varepsilon-\alpha)(\omega-\delta)) n_{0}} e^{-2 \alpha \delta} e^{2(\varepsilon-\alpha)\left(\left(n_{0}+1\right) \omega-\left(n_{0} \omega+\delta\right)\right)} \\
& =V(x(0)) e^{(-2 \alpha \delta+2(\varepsilon-\alpha)(\omega-\delta))\left(n_{0}+1\right)} \\
& =V(x(0)) e^{\frac{(-2 \alpha \delta+2(\varepsilon-\alpha)(\omega-\delta))\left(n_{0}+1\right) \omega}{\omega}} \\
& \leq V(x(0)) e^{\frac{(-2 \alpha \delta+2(\varepsilon-\alpha)(\omega-\delta)) t}{\omega}}
\end{aligned}
$$

Case 2. For $n_{0} \omega \leq t \leq n_{0} \omega+\delta$, using condition (37), we have

$$
V(x(t)) \leq V\left(x\left(n_{0} \omega\right)\right) e^{-2 \alpha\left(t-n_{0} \omega\right)}
$$




$$
\begin{aligned}
& \leq V(x(0)) e^{(-2 \alpha \delta+2(\varepsilon-\alpha)(\omega-\delta)) n_{0}} e^{-2 \alpha\left(t-n_{0} \omega\right)} \\
& \leq V(x(0)) e^{(-2 \alpha \delta+2(\varepsilon-\alpha)(\omega-\delta)) n_{0}} \\
& =V(x(0)) e^{-(-2 \alpha \delta+2(\varepsilon-\alpha)(\omega-\delta))} e^{(-2 \alpha \delta+2(\varepsilon-\alpha)(\omega-\delta))\left(n_{0}+1\right)} \\
& =V(x(0)) e^{-(-2 \alpha \delta+2(\varepsilon-\alpha)(\omega-\delta))} e^{\frac{(-2 \alpha \delta+2(\varepsilon-\alpha)(\omega-\delta))\left(n_{0}+1\right) \omega}{\omega}} \\
& \leq V(x(0)) e^{-(-2 \alpha \delta+2(\varepsilon-\alpha)(\omega-\delta))} e^{\frac{(-2 \alpha \delta+2(\varepsilon-\alpha)(\omega-\delta)) t}{\omega}} .
\end{aligned}
$$

Let $\xi=e^{-(-2 \alpha \delta+2(\varepsilon-\alpha)(\omega-\delta))}$. By (46) and (47), we have

$$
V(x(t)) \leq \xi V(x(0)) e^{\frac{(-2 \alpha \delta+2(\varepsilon-\alpha)(\omega-\delta)) t}{\omega}}, \quad \forall t \geq 0
$$

On the other hand, using the condition (11), we have obtained the following:

$$
\|x(t)\| \leq \sqrt{\frac{\lambda_{2} \xi}{\lambda_{1}}} e^{\frac{(-\alpha \delta+(\varepsilon-\alpha)(\omega-\delta)) t}{\omega}}\|\phi\|, \quad \forall t \geq 0 .
$$

This completes the proof.

Remark 1. In this paper, it is worth pointing out that there were no exponential synchronization for hybrid coupled neural networks with intermittent feedback control. Through a numerical example is given to illustrate the effectiveness of our theoretical results.

\section{Numerical Example}

In this section, we present a simulation example so as to illustrate the usefulness of our main results.

Example 4.1 Consider the system (1) under the controller (3) with the following parameters:

$$
\begin{aligned}
C & =\left[\begin{array}{ll}
3 & 0 \\
0 & 3
\end{array}\right], \quad A=\left[\begin{array}{cc}
0.5 & -0.4 \\
0.4 & 0.5
\end{array}\right], B=\left[\begin{array}{cc}
0.1 & 0.1 \\
0.2 & 0.2
\end{array}\right], \\
D & =\left[\begin{array}{cc}
0.4 & 0 \\
0 & 0.3
\end{array}\right], E=\left[\begin{array}{cc}
0.3 & 0 \\
0.1 & 0.3
\end{array}\right], \quad \Gamma_{1}=\left[\begin{array}{cc}
0.2 & 0 \\
0 & 0.2
\end{array}\right], \\
\Gamma_{2} & =\left[\begin{array}{cc}
0.05 & 0 \\
0 & 0.05
\end{array}\right], \Gamma_{3}=\left[\begin{array}{cc}
0.05 & 0 \\
0 & 0.05
\end{array}\right], \\
\tau_{1} & =0.2, \quad \tau_{2}=1.0, \quad k=1, \quad \sigma=0.02, \quad \mu_{1}=0.3, \quad \mu_{2}=0.2 .
\end{aligned}
$$


The outer-coupling matrix $G^{q}=g_{i j}^{q} \in R^{n \times n},(q=1,2,3)$ are defined as:

$$
\begin{aligned}
g^{1}= & {\left[\begin{array}{ccccc}
-2 & 1 & 0 & 0 & 1 \\
1 & -3 & 1 & 1 & 0 \\
0 & 1 & -2 & 1 & 0 \\
0 & 1 & 1 & -3 & 1 \\
1 & 0 & 0 & 1 & -2
\end{array}\right], } \\
g^{2}=g^{3}= & {\left[\begin{array}{ccccc}
-4 & 1 & 1 & 1 & 1 \\
1 & -4 & 1 & 1 & 1 \\
1 & 1 & -4 & 1 & 1 \\
1 & 1 & 1 & -4 & 1 \\
1 & 1 & 1 & 1 & -4
\end{array}\right] . }
\end{aligned}
$$

Take the activation function as follows:

$$
\begin{aligned}
f_{1}\left(x_{i 1}(t)\right) & =0.5\left(\left|x_{i 1}(t)+1\right|-\left|x_{i 1}(t)-1\right|\right), \\
f_{2}\left(x_{i 2}(t)\right) & =0.25\left(\left|x_{i 2}(t)+1\right|-\left|x_{i 2}(t)-1\right|\right), \\
g_{1}\left(x_{i 1}(t)\right) & =0.5\left(\left|x_{i 1}(t)+1\right|-\left|x_{i 1}(t)-1\right|\right), \\
g_{2}\left(x_{i 2}(t)\right) & =0.25\left(\left|x_{i 2}(t)+1\right|-\left|x_{i 2}(t)-1\right|\right), \\
h_{1}\left(x_{i 1}(s)\right) & =\tanh \left(-x_{i 1}(s)\right), h_{2}\left(x_{i 2}(s)\right)=\tanh \left(-0.8 x_{i 2}(s)\right) .
\end{aligned}
$$

It is easy to see

$$
\begin{array}{rlrl}
F_{1} & =\left[\begin{array}{cc}
-1 & 0 \\
0 & -0.25
\end{array}\right], F_{2}=\left[\begin{array}{ll}
0 & 0 \\
0 & 0
\end{array}\right], J_{1}=\left[\begin{array}{cc}
-1 & 0 \\
0 & -0.25
\end{array}\right], \\
J_{2} & =\left[\begin{array}{ll}
0 & 0 \\
0 & 0
\end{array}\right], & L_{1}=\left[\begin{array}{ll}
0 & 0 \\
0 & 0
\end{array}\right], L_{2}=\left[\begin{array}{cc}
-0.5 & 0 \\
0 & -0.4
\end{array}\right] .
\end{array}
$$

By applying the MATLAB LMI Toolbox, Theorem 2 can be solved to yield the following feasible solutions. Then, the controller gain are

$$
K=\left[\begin{array}{cc}
-0.0196 & 0.0021 \\
0.0026 & -0.0202
\end{array}\right]
$$

Therefore, it follows from Theorem 2 that the system (1) under controller (3) with given parameters is exponentially synchronized, which is further verified by the simulation result of the synchronization error given in Figure 1.

Example 4.2 Consider the system (1) under the controller (4) with the following parameters:

$$
C=\left[\begin{array}{ll}
4 & 0 \\
0 & 3
\end{array}\right], A=\left[\begin{array}{ll}
0.8 & -0.2 \\
0.2 & -0.5
\end{array}\right], B=\left[\begin{array}{ll}
0.5 & 0.5 \\
0.2 & 0.2
\end{array}\right],
$$



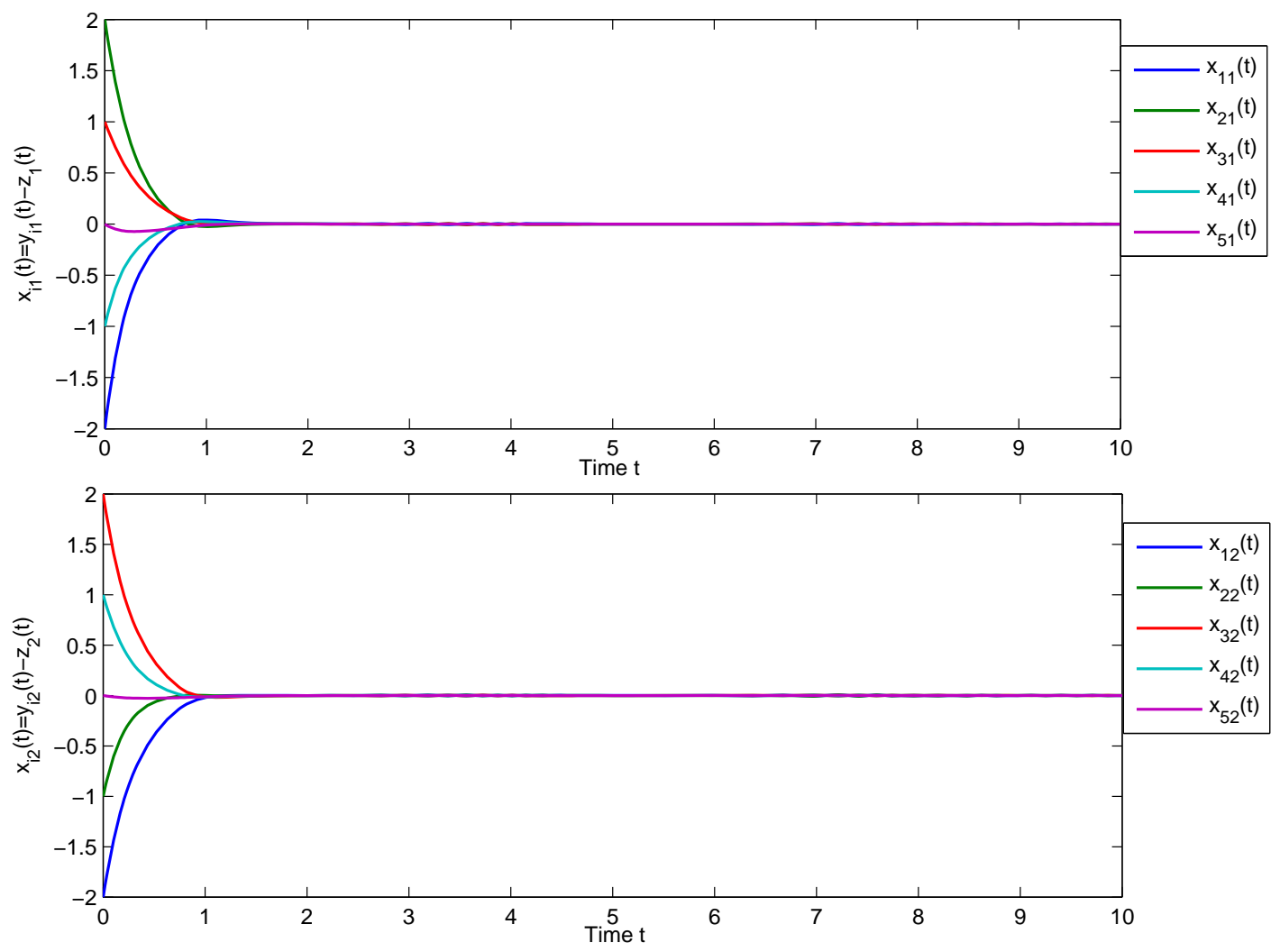

Figure 1: shows the synchronization errors between states $x_{i}(t)$ and isolate node $\widehat{y}(t)$

$$
\begin{aligned}
D & =\left[\begin{array}{cc}
0.1 & 0 \\
0 & 0.1
\end{array}\right], \quad E=\left[\begin{array}{ll}
1 & 0 \\
0 & 1
\end{array}\right], \Gamma_{1}=\left[\begin{array}{cc}
0.2 & 0 \\
0 & 0.2
\end{array}\right], \\
\Gamma_{2} & =\left[\begin{array}{cc}
0.1 & 0 \\
0 & 0.1
\end{array}\right], \Gamma_{3}=\left[\begin{array}{cc}
0.1 & 0 \\
0 & 0.1
\end{array}\right], \\
\tau_{1} & =0.3, \quad \tau_{2}=1.0, \quad k=1, \quad \sigma=0.02, \quad \mu_{1}=0.3, \quad \mu_{2}=0.2, \\
\alpha & =0.2, \quad \delta=0.5, \quad \varepsilon=0.3, \quad \omega=1.0 .
\end{aligned}
$$


The outer-coupling matrix $G^{q}=g_{i j}^{q} \in R^{n \times n},(q=1,2,3)$ are defined as:

$$
\begin{aligned}
g^{1}=g^{2} & =\left[\begin{array}{ccccc}
-2 & 1 & 0 & 0 & 1 \\
1 & -3 & 1 & 1 & 0 \\
0 & 1 & -2 & 1 & 0 \\
0 & 1 & 1 & -3 & 1 \\
1 & 0 & 0 & 1 & -2
\end{array}\right], \\
g^{3} & =\left[\begin{array}{ccccc}
-4 & 1 & 1 & 1 & 1 \\
1 & -4 & 1 & 1 & 1 \\
1 & 1 & -4 & 1 & 1 \\
1 & 1 & 1 & -4 & 1 \\
1 & 1 & 1 & 1 & -4
\end{array}\right]
\end{aligned}
$$

Take the activation function as follows:

$$
\begin{aligned}
f_{1}\left(x_{i 1}(t)\right) & =0.5\left(\left|x_{i 1}(t)+1\right|-\left|x_{i 1}(t)-1\right|\right) \\
f_{2}\left(x_{i 2}(t)\right) & =0.25\left(\left|x_{i 2}(t)+1\right|-\left|x_{i 2}(t)-1\right|\right) \\
g_{1}\left(x_{i 1}(t)\right) & =0.25\left(\left|x_{i 1}(t)+1\right|-\left|x_{i 1}(t)-1\right|\right) \\
g_{2}\left(x_{i 2}(t)\right) & =0.5\left(\left|x_{i 2}(t)+1\right|-\left|x_{i 2}(t)-1\right|\right), \\
h_{1}\left(x_{i 1}(s)\right) & =\tanh \left(-1.2 x_{i 1}(s)\right), h_{2}\left(x_{i 2}(s)\right)=\tanh \left(-0.8 x_{i 2}(s)\right) .
\end{aligned}
$$

It is easy to see

$$
\begin{array}{llrl}
F_{1} & =\left[\begin{array}{cc}
-1 & 0 \\
0 & -0.25
\end{array}\right], F_{2}=\left[\begin{array}{ll}
0 & 0 \\
0 & 0
\end{array}\right], J_{1}=\left[\begin{array}{cc}
-0.25 & 0 \\
0 & -1
\end{array}\right], \\
J_{2} & =\left[\begin{array}{ll}
0 & 0 \\
0 & 0
\end{array}\right], & L_{1}=\left[\begin{array}{ll}
0 & 0 \\
0 & 0
\end{array}\right], L_{2}=\left[\begin{array}{cc}
-0.6 & 0 \\
0 & -0.4
\end{array}\right] .
\end{array}
$$

By applying the MATLAB LMI Toolbox, Theorem 3 can be solved to yield the following feasible solutions. Then, the controller gain are

$$
K=\left[\begin{array}{cc}
-0.0126 & 0.0030 \\
0.0030 & -0.0149
\end{array}\right]
$$

Therefore, it follows from Theorem 3 that the system (1) under controller (4) with given parameters is exponentially synchronized, which is further verified by the simulation result of the synchronization error given in Figure 3-4. 

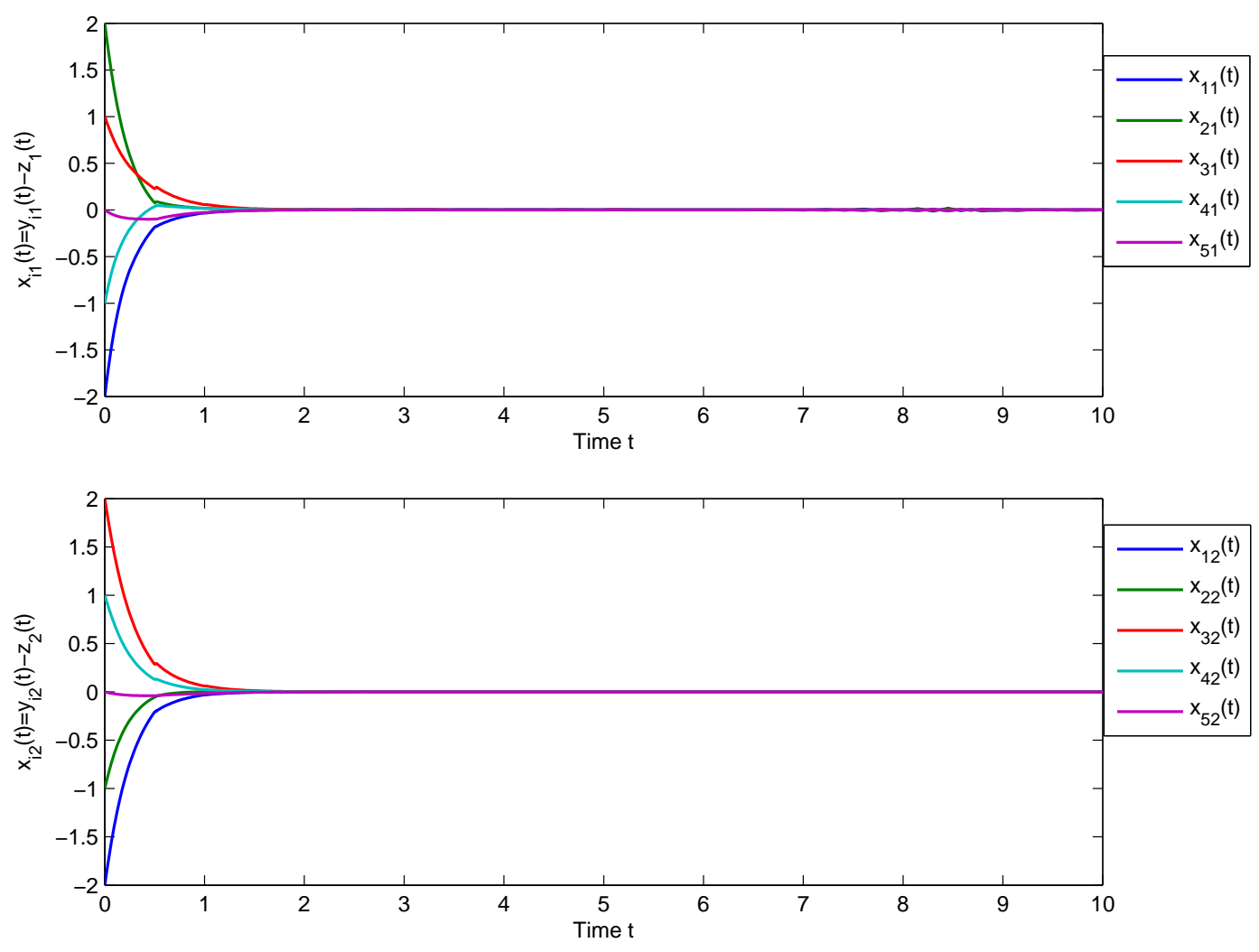

Figure 2: shows the synchronization errors between states $x_{i}(t)$ and isolate node $\widehat{y}(t)$

\section{Conclusions}

In this paper, we have investigated the exponential synchronization of neural networks with simultaneous presence of time delays and leakage delay. The designed controller ensures the exponential synchronization of hybrid couple neural networks are proposed via intermittent feedback control. The provided conditions are expressed in terms of linear matrix inequality by constructing a set of improved Lyapunov-Krasovskii functionals and Kronecker product properties, Numerical examples illustrate the effectiveness of the results. 


\section{Acknowledgments}

The first author is supported by the Graduate School, Chiang Mai University. The third author is supported by the Centre of Excellence in Mathematics, the Commission on Higher Education, Thailand.

\section{References}

[1] L. M. Pecora and T. L. Carroll, Synchronization in chaotic systems, Phys. Rev. Lett., 64 (1990), 821-824.

[2] A. Cichocki, R. Unbehauen, Neural Networks for Optimization and Signal Processing, Wiley, Hoboken, NJ, 1993.

[3] J. Liang and J. Cao, Global asymptotic stability of bi-directional associative memory networks with distributed delays, Appl. Math. Comput., 152 (2004), 415-424.

[4] S. Cai, Q. He, J. Hao and Z. Liu, Exponential synchronization of complex networks with nonidentical time-delayed dynamical nodes, Physics Letters A. 374 (2010), 2539-255.

[5] J. Lu, Daniel W.C. Ho and Ming Liu, Globally exponential synchronization in an array of asymmetric coupled neural networks, Physics Letters A, $\mathbf{3 6 9}$ (2007) 444-451.

[6] D. Gong, H. Zhang, Z. Wang and B. Huang, Novel synchronization analysis for complex networks with hybrid coupling by handling multitude Kronecker product terms, Neurocomputing, 82 (2012), 14-20.

[7] H. Zhang, D. Gong, Z. Wang and D. Ma, Synchronization Criteria for an Array of Neutral-Type Neural Networks with Hybrid Coupling: A Novel Analysis Approach, Neural Process Lett, 35 (2011), 29-45.

[8] J. Cao, G. Chen and P. Li, Global synchronization in an array of delayed neural networks with hybrid coupling, IEEE Transactions on Systems, Man and Cybernetics, Part B (Cybernetics), 38(2) (2008), 488-498.

[9] J. Cao and L. Li, Cluster synchronization in an array of hybrid coupled neural networks with delay, Neural Networks, 22 (2009), 335-342. 
[10] W. Wang and J. Cao, Synchronization in an array of linearly coupled networks with time-varying delay, Physica A, 366 (2006), 197-211.

[11] K. Gu, V.L. Kharitonov and J.Chen, Stability of time-delay system, Boston: Birkhauser, 2003.

[12] Y. Wang, H. Zhang, X. Wang and D. Yang, Networked Synchronization Control of Coupled Dynamic Networks With Time-Varying Delay, IEEE, 40 (2010), 1468-1479.

[13] M.J. Park, O.M. Kwon,Ju H. Park, S.M. Lee and E.J. Cha, Synchronization criteria for coupled neural networks with interval time-varying delays and leakage delay, Appl. Math. Comput., 218 (2012), 6762-6775.

[14] M.J. Park, O.M. Kwon, Ju H. Park, S.M. Lee and E.J. Cha, Synchronization criteria for coupled stochastic neural networks with time-varying delays and leakage delay, Journal of the Franklin Institute, 349 (2012), 1699-1720.

[15] Q. Gan and Y. Liang, Synchronization of chaotic neural networks with time delay in the leakage term and parametric uncertainties based on sampled-data control, Journal of the Franklin Institute, 349 (2012), 19551971.

[16] N. Li, J. Hu, J.Hu and L. Li, Exponential state estimation for delayed recurrent neural networks with sampled-data, Nonlinear Dyn, 69 (2012), $555-564$.

[17] T. Botmart and P. Niamsup, Adaptive control and synchronization perturbed Chuas system, Math. Comput. Simulation, 75 (2007), 37-55.

[18] T. Botmart, P. Niamsup and X. Liu, Synchronization of non-autonomous chaotic systems with time-varying delay via delayed feedback control, Commun. Nonlinea.r Sci. Numer. Simulat., 17 (2012), 1894-1907.

[19] H. Zhu and B. Cui, Stabilization and synchronization of chaotic systems via intermittent control, Commun. Nonlinea.r Sci. Numer. Simulat., 15 (2010), 3577-3586.

[20] Zochowski M, Intermittent dynamical control, Phys D., 145 (2000), 18190. 
[21] X. Liu, X. Shen and H. Zhang, Intermittent Impulsive Synchronization of Chaotic Delayed Neural Networks Differ Equ Dyn Syst.,19 (2011), 149 169.

[22] J. Xing, H. Jiang and C. Hu, Exponential synchronization for delayed recurrent neural networks via periodically intermittent control, Neurocomputing., 113 (2013), 122-129.

[23] T. Huang, C. Li, Chaotic synchronization by the intermittent feedback method, J Comput Appl Math., 234 (2010), 1097-1104. 